\title{
Syntheses and Biological Activity of the HDAC Class I Inhibitor Largazole
}

\author{
Tobias Seiser and Nicolai Cramer*
}

\begin{abstract}
Histone deacetylase inhibitors are an emerging class of antiproliferative agents that have the potential to combat cancer on an epigenetic level. The recently reported marine natural product largazole has been recently isolated in trace amounts from cyanobacteria and displays a very potent and selective antiproliferative activity towards a number of cell lines. Several lines of evidence suggested it might act as a histone deacetylase inhibitor. These compelling properties have generated considerable interest in the synthetic community which resulted in several total syntheses of largazole. The present review offers a brief overview of the synthetic routes and some early structure-activity relationships.
\end{abstract}

Keywords: Anticancer agents $\cdot$ Histone deacetylase $\cdot$ Natural products $\cdot$ Total synthesis

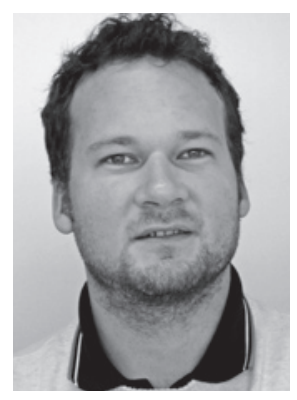

Nicolai Cramer received his diploma in chemistry from the University of Stuttgart in 2003 and obtained his $\mathrm{PhD}$ with Professor Sabine Laschat in 2005. After a research stay at Osaka University with the Professors Michio Murata and Sumihiro Hase, he joined the group of Professor Barry M. Trost at Stanford University as a Humboldt postdoctoral fellow. In fall 2007, he was awarded a Liebig-Fellowship and started his independent career as Habilitant at the ETH Zurich under the mentorship of Professor Erick M. Carreira. His research group focuses on natural product synthesis and transition metal catalysis.

\section{Introduction}

Histone deacetylase enzymes (HDAC) are classes of enzymes that modify the chromatine and are responsible for the regulation of the gene transcription in eukaryotic cells. The histone deacetylases cleave $N$-acetyl groups on the terminal lysine moieties of the core histones. This results in tighter binding of the DNA to the histones due to increased Coulomb attraction of the negatively charged DNA and the positively charged ammonium residues of the histones, thus impeding the access of many transcriptional factors to the DNA. As consequence, the expression of the target genes is repressed. Several lines of evidence suggest that aberrant histone acetylation plays a causative role in oncogenesis. ${ }^{[1]}$ The HDACs are $\mathrm{Zn}^{2+}$ based metalloenzymes and 18 different HDACs, divided into four classes, have been identified to date. ${ }^{[2]}$ The clinical relevance of the HDAC Class I for cancer chemotherapy has been recognized and their selective inhibition holds promise for cancer treatment on an epigenetic level. ${ }^{[3]}$
Exposure of cancer cells to HDAC inhibitors has been associated with several effects such as growth arrest and cell differentiation, induction of apoptosis and inhibition of angiogenesis. ${ }^{[4]}$ Additionally to the effects directly seen with HDAC inhibitors alone, these compounds are believed to synergize or potentiate with other cytotoxic agents. ${ }^{[5]}$ The side effects and the undesired toxicity of the first generation of HDAC inhibitors may be attributed to their unselective inhibition of all HDAC classes. Hence, the identification of more class- or even isoform selective inhibitors has become a significant research area.

\section{Depsipeptides with HDAC Inhibitory Activities}

Several natural products such as trapoxin, FK228, FR901375 and spiruchostatin, discovered over the past few years, exhibit potent HDAC inhibitory activity (Scheme 1). ${ }^{6]}$ It was shown that these bi-
${ }^{*}$ Correspondence: Dr. N. Cramer

Laboratorium für Organische Chemie ETH Zürich

$\mathrm{HCl} \mathrm{H} 304$

Wolfgang-Pauli-Strasse 10

$\mathrm{CH}-8093$ Zürich

Tel.: + 41446326412

Fax: + 41446321328

E-mail: nicolai.cramer@org.chem.ethz.ch
Scheme 1.

Depsipeptides having HDAC inhibitory activity and the activation mode of FK228.

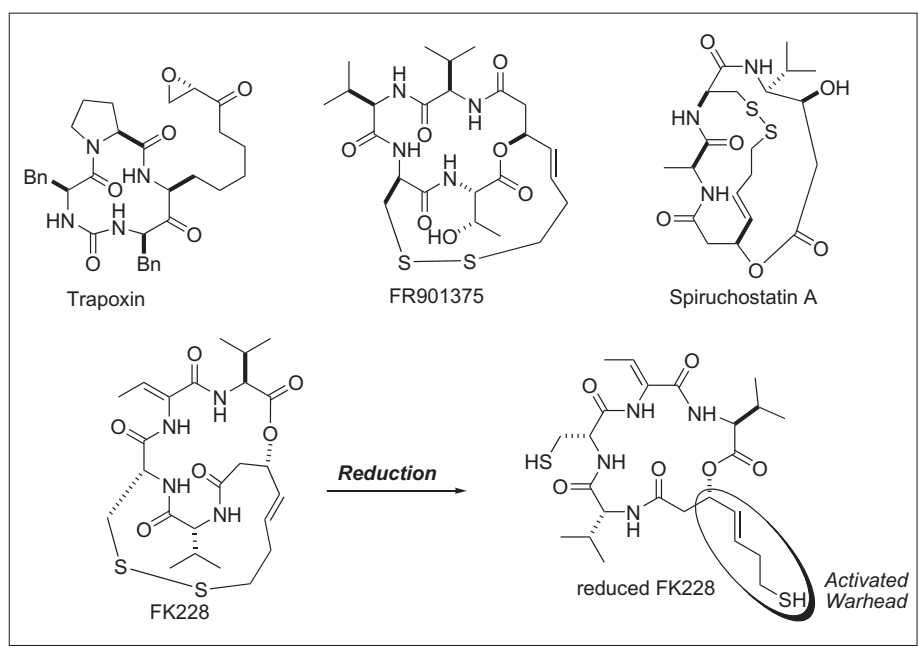




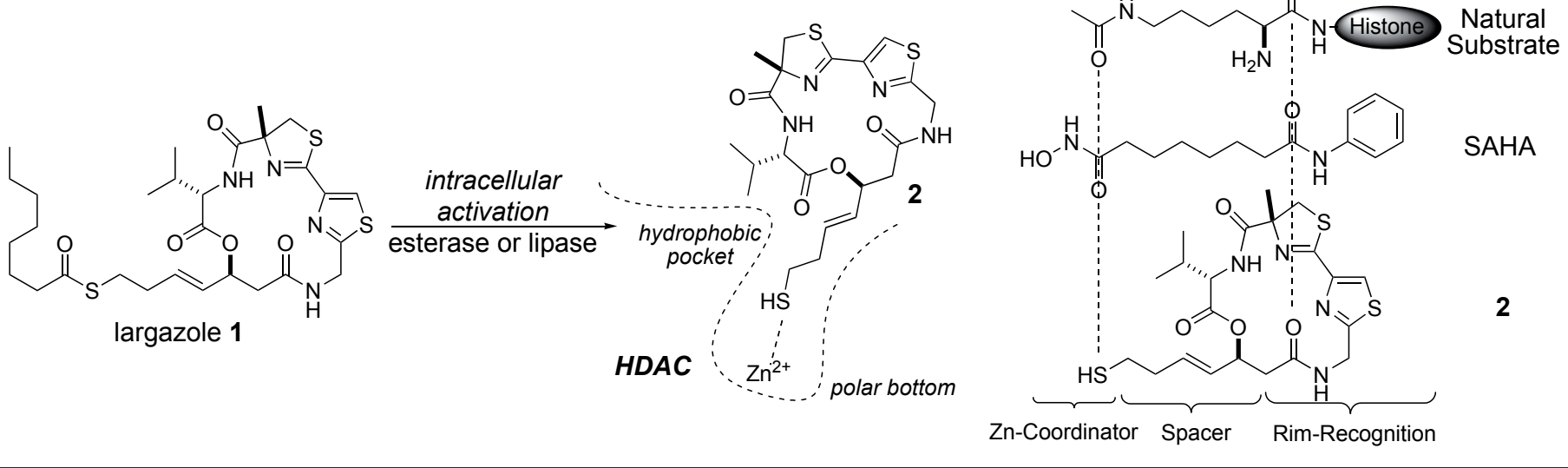

Scheme 2. Activation and plausible binding mode of largazole.

cyclic bisulfides are pro-drugs which are intracellular activated via reductive cleavage of their bisulfide moiety to reveal the free thiols as activated warheads (reduced FK228).[7] Consistent with the data obtained from co-crystallization experiments of HDAC-like proteins and first-generation HDAC inhibitors such as SAHA (suberoylanilide hydroxamic acid), ${ }^{[8]}$ it is most probable that these inhibitors mimic the natural substrate and that the free thiol chelates the zinc ion residing in the $11 \AA$ deep tubular pocket of the HDAC protein. A potentially successful HDAC inhibitor has a $\mathrm{Zn}^{2+}$-binding arm, consisting of a suitable zinc chelating group and a spacer, attached to an appropriate template responsible for the selective rim recognition of the different HDAC isoforms (Scheme 2). While SAHA, marketed by Merck as Zolinza, has been recently approved by the FDA, ${ }^{\left[{ }^{8 a}\right]}$ FK 228 is still undergoing phase II clinical trials. ${ }^{[9]}$

\section{Largazole, a Potent Antiproliferative Marine Natural Product}

Luesch and coworkers disclosed in the beginning of 2008 the structure of largazole (1), which is a novel peptidepolyketite hybride. ${ }^{[10]}$ It was isolated in trace amounts from a marine cyanobacterium of the genus Symploca collected from Key Largo, Florida. Largazole displays very potent, yet up to 10 -fold selective, differential growth inhibition activity in several transformed human and murinederived cell lines compared to their nontransformed counterparts. This remarkable selectivity against the cancer cells sparked the interest of several research groups in the elucidation of its molecular target and mode of action. It seemed plausible that in analogy to FK228, largazole is a pro-drug that would be intracellularly activated by a hydrolytic cleavage of the thioester moiety by lipases and esterases. The resulting largazole-thiol (2) can subsequently bind to the $\mathrm{Zn}^{2+}$ of the HDAC (Scheme 2). Recent intense research activities in this area not only culminated in syntheses by the groups of Hong, Cramer, Williams, Ye, Doi, Phillips and Ghosh, but also generated a solid body of work on the biological properties of $1^{[11]}$

\section{Synthetic Routes to Largazole}

Largazole's interesting yet chemically tractable structure consists of a $\alpha$-methylcysteine-derived thiazoline fused to a thiazole embedded within a $16-\mathrm{mem}-$ bered lactone and a rarely in natural products occurring thioester. One of the points to address for a synthesis consisted in the manipulation of the somewhat labile thioester moiety of $\mathbf{1}$. Two approaches were developed: An introduction of the sulfhydryl group, protected as trityl or bisulfide, at an early stage of the synthesis and its late stage elaboration into the thioester (Scheme 3 ). The groups of Cramer, Hong and Phillips $^{[11]}$ followed a second approach introducing the complete thioester side chain in the very last step of the synthesis via a cross metathesis reaction, thus avoiding several protection group manipulations. An additional strategic advantage consists in the facile generation of analogues by simple exchange of the olefin cross metathesis partner. However, there were doubts about the feasibility of the metathesis reaction with olefins having sulfur substituents in a critical chelating position such as $\mathbf{3}$. The second key disconnection consisted in the lactamization to form the cyclic template of largazole. Two sites ( $\boldsymbol{A}$ and $\boldsymbol{B}$ ) have been identified and successfully utilized for the critical ring closure (Scheme 3).

Most groups chose to construct the oxygenated stereogenic center in $\beta$-position to the ester of $\mathbf{6 b}$ by a Nagao aldol reaction ${ }^{[12]}$ whereas Cramer and Phillips accessed 6a via an enzymatic resolution of the racemic aldol adduct of acrolein and tert-butyl

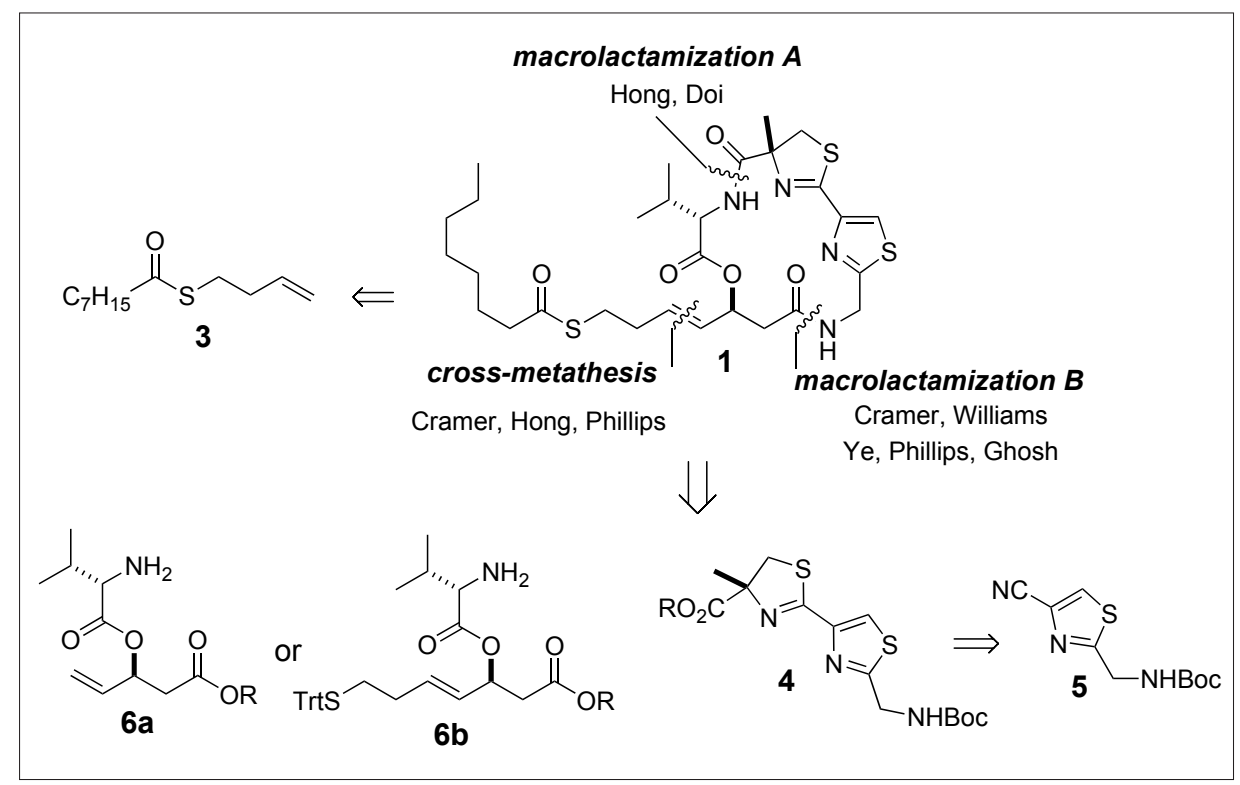

Scheme 3. Synthetic strategies and key building blocks. 


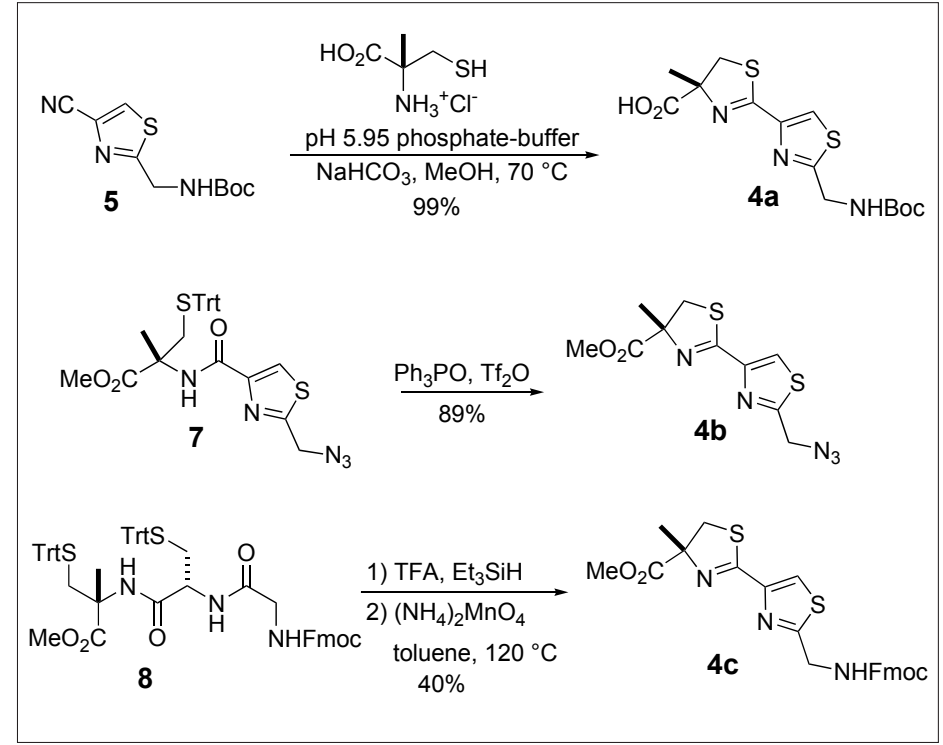

Scheme 4. Syntheses of the thiazolthiazoline building block 4.

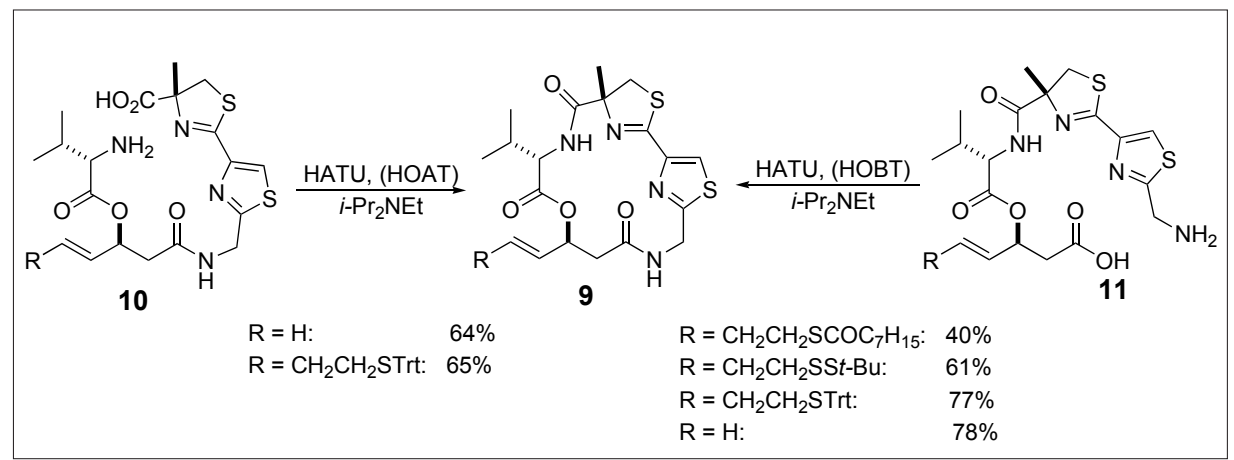

Scheme 5. Two strategies proved to be successful to close the macrocycle.

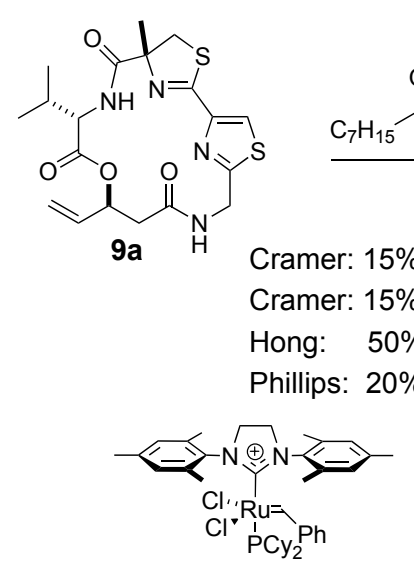

Grubbs-II (12)

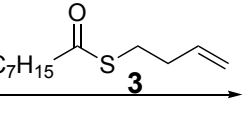

14, DCE, $80{ }^{\circ} \mathrm{C}$,

3, DCE, $80^{\circ} \mathrm{C}$,

$44 \%$

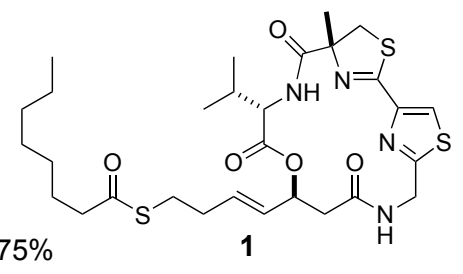

$41 \%$

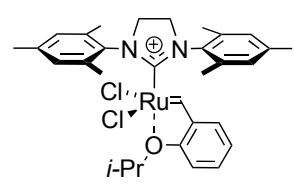

Hoveyda-Grubbs-II (13)

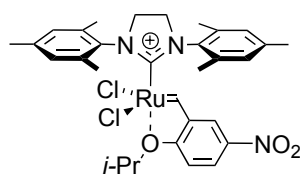

Grela catalyst (14)

Scheme 6. Addressing the pivotal cross-metathesis.

acetate $^{[13]}$ followed by esterification. The thiazole-thiazoline building block $\mathbf{4}$ was assembled from a functionalized thiazole $5^{[14]}$ and $(R)$ - $\alpha$-methyl cysteine (Scheme $4)$. (R)- $\alpha$-methyl cysteine itself is conveniently prepared from $(R)$-cysteine by the Pattenden modification of the Seebach protocol.[15] Heating a nitrile and an ami- nothiol under basic conditions, e.g. with triethylamine in methanol usually leads to a clean reaction to the corresponding thiazoline. However, it is known that sterically more demanding aminothiols, e.g. $\alpha$-methylcysteine, react relatively sluggish, need long reaction times and give moderate yields. It was found that such shortcomings can be overcome by the usage of mild aqueous conditions ( $\mathrm{pH}=5.9$ phosphate buffer/ $\mathrm{NaHCO}_{3}$ in refluxing methanol) providing the thiazoline $\mathbf{4 a}$ in an excellent yield. ${ }^{[16]}$ As an alternative to this condensation, Doi and Ghosh prepared the thiazoline $\mathbf{4 b}$ from the corresponding amide 7 under Kelly's conditions ${ }^{[17]}$ using $\mathrm{Ph}_{3} \mathrm{PO}$ and $\mathrm{Tf}_{2} \mathrm{O}$. Doi demonstrated additionally a remarkable tandem dehydrative cyclization of the protected tripeptide $\mathbf{8}$. Cleavage of both trityl groups of $\mathbf{8}$, followed by treatment with a catalytic amount of $\left(\mathrm{NH}_{4}\right)_{2} \mathrm{MnO}_{4}$ under Dean-Stark conditions afforded thiazolinethiazole $\mathbf{4 c}$ in $40 \%$ yield.

The amide bond formation linking the two core fragments $\mathbf{4}$ and $\mathbf{6}$ was straightforward under standard conditions setting the stage for the cyclization. Subsequently, different reagents were screened to perform the crucial lactamization. In consensus, the use of HATU as activation reagent with or without HOBT/HOAT provided, regardless of the cyclization site, the desired lactam in 40-78\% yield depending on the substitution pattern (Scheme 5).

Having already installed the sulfhydryl group at an early stage, Williams, Ye and Doi ${ }^{[11 c-e]}$ completed their syntheses by the introduction of the thioester by a two step deprotection-acylation sequence. Cramer, Hong and Phillips ${ }^{[11 \mathrm{a}, \mathrm{b}, \mathrm{f}]}$ took the challenge in installing the side chain via a late stage cross-metathesis. All three groups had to address the interference of the coordinating thioester or its degradation products with the ruthenium metathesis catalyst. This inherent difficulty is reflected by the required high catalyst loading (20-50 mol\%) and the modest yields obtained with the Grubbs-II (12) or Hoveyda-Grubbs-II (13) catalyst (Scheme 6).

The best solution consisted in using the more active Grela catalyst (14), a modification of $\mathbf{1 3}$ having a $p$-nitro substituent. [18] With 15 mol\% of $\mathbf{1 4}$ Cramer and coworkers obtained the coupling product largazole in $75 \%$ yield.

In summary, the modular syntheses are reasonably short (9-19 linear steps), high yielding (up to $22 \%$ overall yield) and rely on ready accessible building blocks. Several of the illustrated routes have the proven potential to provide largazole in gram quantities and subsequent biological studies should therefore not be limited by any material shortages.

\section{Activity Profile of Largazole Analogues}

The potent antiproliferative activity of 1 was confirmed by several groups and the mode of action of the free thiol 2 was experimentally established as a HDAC inhibitor.[11a,b,c,e,f] In more detailed stud- 
ies, Williams ${ }^{[11 c]}$ showed that $\mathbf{2}$ is a potent HDAC Class-I inhibitor with unprecedented selectivity for HDAC 1, HDAC 2 and HDAC 3. They reported in enzymatic assays a $K_{\mathrm{i}}$ value of $70 \mathrm{pM}$ for HDAC 1 and HDAC 2, which is about twice as potent as FK228 (140 pM) and more than 140-fold as SAHA (Zolinza, Merck Research Laboratories). Along with the preparation of synthetic largazole, several groups used their approaches to synthesize different analogues giving deeper insights in the structure activity relationship (Fig.). ${ }^{[1,19]}$ Hong and coworkers reported that a minor modification of the cyclic core template, exchanging valine for alanine, has a moderate impact and decreases the activity three-fold. ${ }^{[19]}$ However, inverting the stereochemistry of the allylic hydroxyl group resulted in a complete loss of activity. Noncyclic synthetic intermediates or the terminal alkene 9a displayed no activity either. The presence of a latent sulfhydryl group (thioester or bisulfide), cleavable under physiological conditions, or the free thiol itself is mandatory for activity. Replacement of the thioester by a carbon chain, ketone, alcohol, or ester analogue led to completely inactive compounds. ${ }^{[11 b, f, 19]}$ This is in accordance with their inability to properly chelate the zinc ion in the HDAC enzyme. Furthermore, the groups of Cramer and Hong reported both that the length of the spacer is essential for potent antiproliferative activity. ${ }^{[11 b, 19]}$ Any alteration of the four carbon thiobutenyl spacer, either an extension or a truncation, dramatically decreases the potency by at least several orders of magnitude.

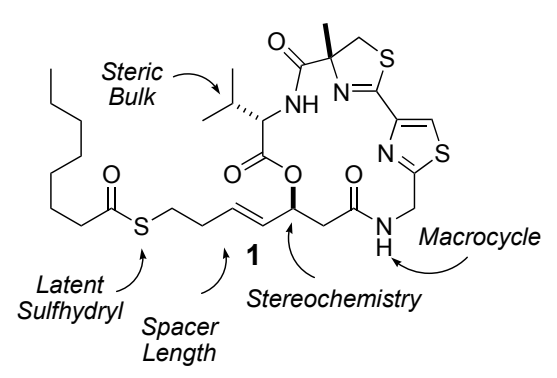

Fig. Initial SAR studies: Relevant factors to preserve activity.

This finding underlines the importance of the right spatial distance of the sulfhydryl group to the cyclic core. It seems that there is only a narrow window for optimal $\mathrm{Zn}^{2+}$ coordination. Although the free thiol $\mathbf{2}$ has been identified as the active agent, it displays reduced potency in cell-based assays compared to the parent thioester 1. Williams and coworkers reported for a panel of human malignant melanoma cells $\mathrm{IC}_{50}$ values between $45-315 \mathrm{nM}$ for 1 and 360-2600 $\mathrm{nM}$ for $2,{ }^{[11 \mathrm{c}]}$ which are in good agreement with the results of other published data. ${ }^{[111 b, e, f]}$ The lower potency of $\mathbf{2}$ is attributed to its increased polarity which decreases cellular uptake. On the other hand, modifications of the capping group do not have a big impact on the potency. It has been shown that the octanoic acid moiety can be replaced by other carboxylic acids and also by a bisulfide, while the complete activity was maintained. The protective octanoic acid cap proved to be beneficial for two reasons: First, cellular uptake is facilitated as the more hydrophobic nature of the thioester allows a more facile penetration of the cell membrane compared to the thiol. Secondly, the capped molecule is significantly more robust and the free thiol is only presented in the inner of the cell. There is a consensus that the sulfhydryl group represents a very convenient handle for novel and innovative protect and release strategies. For example, conjugating the thiol to an appropriate antibody or loading it onto self-immolative constructs $^{[20]}$ to specifically target predefined areas or tissues is imaginable.

\section{Conclusion}

In a very limited time frame, several viable and competitive synthetic routes to the natural product largazole have been devised. Studies along these lines revealed that it is a pro-drug which is hydrolytically activated to give the active free thiol. The combination of the protective cap and the zinc binding abilities of the free thiol result in the most potent and selective HDAC inhibitor disclosed to date. After this first rush, there are ample opportunities to design and synthesize more sophisticated agents targeting the inhibition of specific HDACs and to further exploit the cancer therapeutic potential of the cyclic template of largazole.

\section{Acknowledgements}

We thank the Swiss National Foundation (21-119750.01) and Prof. Dr. E. M. Carreira for generous financial support. N. C. is grateful to the Fonds der Chemischen Industrie for a Liebig-Fellowship.

Received: December 10, 2008

[1] a) A. H. Lund, M. van Lohuizen, Genes Dev. 2004, 18, 2315; b) S. B. Baylin, J. E. Ohm, Nature Rev. Cancer 2006, 6, 107.

[2] R. W. Johnstone, Nature Rev. Drug Discovery 2002, 1, 287.

[3] J. E. Bolden, M. J. Peart, R. W. Johnstone, Nature Rev. Drug Discovery 2006, 5, 769.

[4] a) Y. G. Zheng, J. Wu, Z. Chen, M. Goodman, Med. Res. Rev. 2008, 28, 645; b) M.-J. Lee, Y. S. Kim, S. Kummar, G. Giaccone, J. B. Trepel, Curr. Opin. Oncol. 2008, 20, 639; c) X.-J. Yang, E. Seto, Oncogene 2007, 26, 5310.

[5] a) H. J. Kwon, M. S. Kim, M. J. Kim, H. Nakajima, K.-W. Kim, Int. J. Cancer 2002,
97, 290; b) P. A. Marks, V. M. Richon, R. A. Rifkind, J. Natl. Cancer Inst. 2000, 92, 1210; c) C.-Y. Gui, L. Ngo, W. S. Xu, V. M. Richon, P. A. Marks, Proc. Natl. Acad. U.S.A. 2004, 101, 1241.

[6] a) P. A. Townsend, S. J. Crabb, S. M. Davidson, P. W. M. Johnson, G. Packham, A. Ganesan, 'The bicyclic depsipeptide family of histone deacetylase inhibitors', in 'Chemical Biology', Eds. S. L. Schreiber, T. M. Kapoor, G. Wess, Wiley-VCH, Weinheim, 2007; Vol. 69, pp 3-720; b) M. Kijima, M. Yoshida, K. Sugita, S. Horinouchi, T. Beppu, J. Biol. Chem. 1993, 268 , 22429.

[7] R. Furumai, A. Matsuyama, N. Kobashi, K.-H Lee, M. Nishiyama, H. Nakajima, A. Tanaka, Y. Komatsu, N. Nishino, M. Yoshida, S. Horinouchi, Cancer Res. 2002, 62, 4916.

[8] a) P. A. Marks, R. Breslow, Nat. Biotechnol. 2007, 25, 84; b) T. K. Nielsen, C. Hildmann, A. Dickmanns, A. Schwienhorst, R. Ficner, J. Mol. Biol. 2005, 354, 107.

[9] R. L. Piekarz, R. A. Frye, J. J. Wright, S. M. Steinberg, D. J. Liewehr, D. R. Rosing, V. Sachdev, T. Fojo, S. E. Bates, Clin. Cancer Res. 2006, 12, 3762.

[10] K. Taori, V. J. Paul, H. Luesch, J. Am. Chem. Soc. 2008, 130, 1806.

[11] In the order of submission: a) Y. Ying, K. Taori, H. Kim, J. Hong, H. Luesch, J. Am. Chem. Soc. 2008, 130, 8455; b) T. Seiser, F. Kamena, N. Cramer, Angew. Chem. Int. Ed. 2008, 120, 6483 ; c) A. Bowers, N. West, J. Taunton, S. L. Schreiber, J. E. Bradner, R. M. Williams, J. Am. Chem. Soc. 2008, 130, 11219; d) Q. Ren, L. Dai, H. Zhang, W. Tan, Z. Xu, T. Ye, Synlett 2008, 2379; e) Y. Numajiri, T. Takahashi, M. Takagi, K. Shin-ya, T. Doi, Synlett 2008, 2483; f) C. G. Nasveschuk, D. Ungermannova, X. Liu, A. J. Phillips, Org. Lett. 2008, 10, 3595; g) A. K. Ghosh, S. Kulkarni, Org. Lett. 2008, 10, 3907.

[12] Y. Nagao, Y. Hagiwara, T. Kumagai, M. Ochiai, T. Inoue, K. Hashimoto, E. Fujita, J. Org. Chem. 1986, $51,2391$.

[13] a) S. Vrielynck, M. Vandewalle, A. M. Garcia, J. L. Mascarenas, A. Mourino Tetrahedron Lett. 1995, 36, 9023; b) C.-H. Tan, T. Stork, N. Feeder, A. B. Holmes, Tetrahedron Lett. 1999, 40, 1397.

[14] M. Knopp, S. Koser, B. Schäfer (BASF AG), DE-19934066A1, 2001.

[15] a) A. Jeanguenat, D. Seebach, J. Chem. Soc. Perkin Trans 1 1991, 2291; b) G. Pattenden, S. M. Thorn, M. F. Jones, Tetrahedron 1993, 49, 21318.

[16] R. J. Bergeron, J. Wiegand, J. S. McManis, B. H. McCosar, W. R. Weimar, G. M. Brittenham, R. E. Smith, J. Med. Chem. 1999, 42, 2432.

[17] S.-L. You, H. Razavi, J. W. Kelly, Angew. Chem. Int. Ed. 2003, 42, 83.

[18] A. Michrowska, R. Bujok, S. Harutyunyan, V. Sashuk, G. Dolgonos, K. Grela, J. Am. Chem. Soc. 2004, 126, 9318.

[19] Y. Ying, Y. Liu, S. R. Byeon, H. Kim, H. Luesch, J. Hong, Org. Lett. 2008, 10, 4021.

[20] D. Shabat, J. Polym. Sci., Part A: Polym. Chem. 2006, 44, 1569 